\title{
MV-pairs and state operators
}

\author{
S. Pulmannová ${ }^{\star \star}$ \\ Mathematical Institute, Slovak Academy of Sciences \\ Štefánikova 49, SK-81473 Bratislava, Slovakia
}

\begin{abstract}
Flaminio and Montagna (2008) enlarged the language of MV-algebras by a unary operation $\sigma$, called internal state or a state operator, equationally defined so as to preserve the basic properties of a state in its usual meaning. The resulting class of MV-algebras is called state MV-algebras. Jenča (2007) and Vetterlein (2008), using different approaches, represented MV-algebras through the quotient of a Boolean algebra $B$ by a suitable subgroup $G$ of the group of all automorphisms of $B$. Such a couple $(B, G)$ is called an MV-pair. We introduce the notion of a state MV-pair as a triple $(B, G, \sigma)$, where $(B, G)$ is an MV-pair and $\sigma$ is a state operator on $B$, and show that there are relations between state $\mathrm{MV}$-pairs and state MV-algebras similar to the relations between MV-pairs and MV-algebras. We also give a characterization of those MV-pairs, resp. state MV-pairs, that induce subdirectly irreducible MV-algebras, resp. state MV-algebras.
\end{abstract}

MV-algebras were introduced as algebraic bases for many-valued logic [3]. That is, MV-algebras stand in relation to the Eukasziewicz infinite valued logic as Boolean algebras stand to classical two-valued logic. A key relationship between Boolean algebras and MV-algebras lies in the fact that the set of all idempotents of an MV-algebra $M$ is a Boolean algebra, in fact the greatest Boolean subalgebra of $M$. The Boolean algebra of idempotents can be considered as a system of classical propositions, while the surrounding algebra $M$ can be considered as an extension of the classical logic by fuzzy, resp. unsharp propositions.

Another relation between MV-algebras and Boolean algebras was described in [10], where a representation theorem for MV-algebras is given in terms of Boolean algebras and their automorphism groups. Actually, it is shown in [10] that given a Boolean algebra $B$ and a subgroup $G$ of its automorphism group satisfying certain conditions, the pair $(B, G)$ can be canonically associated with an MV-algebra. Such pairs $(B, G)$ are called MV-pairs. Conversely, given an MValgebra $M$, if $B(M)$ denotes its R-generated Boolean algebra [11] and $G(M)$ is a special subgroup of the automorphism group of $B(M)$, it turns out that $(B(M), G(M))$ forms an MV-pair. In [7], a categorical development of the results in $[10]$ is presented.

^ This contribution is based on a joint work with E. Vinceková.

** This work was supported by grant VEGA 2/0059/12 and by Science and Technology Assistance Agency under the contract no. APVV-0178-11. 
MV-algebras are equivalent to MV-effect algebras [4], which are a special subclass of a more general class of effect algebras [9]. Equivalence between MValgebras and MV-effect algebras was used in [10], where it was proved that given an MV-pair $(B, G), G$ induces a congruence on $B$ such that the quotient $B / G$ is an MV-effect algebra.

States on MV-algebras, as averaging of truth values, were studied in [12]. Recently, the notion of a state was generalized in [8] to an algebraically defined notion for MV-algebras. The language of MV-algebras has been enlarged by a unary operation $\sigma$, called an internal state or a state operator. Such MV-algebras are called state-MV-algebras. These algebras are now intensively studied, e.g. [6]. In [2], a generalization of the notion of a state operator for effect algebras was introduced and studied. The authors also introduced the notion of a strong state operator, and have shown that the effect algebra state operator coincides, on MV-effect algebras, with the MV-algebra state operator as introduced in [8] if and only if it is strong.

The present contribution is based on [1], where internal states in connection with MV-pairs are studied. Namely, we study relations between state MValgebras and state Boolean algebras, which are connected by an MV-pair. One of the main results is the following [1, Theorem 4.11]:

Theorem 1. If $(B, G, \sigma)$ is a (strong) state- $M V$-pair, then $M=B / G$ is a (strong) state- $M V$-effect algebra with the state operator $\sigma_{*}\left([a]_{G}\right)=[\sigma(a)]_{G}$. Conversely, if $(M, \sigma)$ is a (strong) state- $M V$-effect algebra, then $\left(B(M), G(M), \sigma^{*}\right)$, where $\sigma^{*}(a)=\sigma\left([a]_{G(M)}\right)$, is a strong state-MV-pair and $\left(\sigma^{*}\right)_{*}=\sigma$.

The outline of the talk is as follows.

1. We introduce some definitions and known results that we need in what follows.

2. We introduce a new definition of morphisms of MV-pairs (which, in our opinion is more transparent), and we show that it is equivalent with the original definition introduced in [7].

3. We introduce the notions of state MV-pairs and strong state MV-pairs, and study their relations with state MV-algebras.

4. We find conditions under which an MV-pair, resp. a state MV-pair, gives rise to a linearly ordered and to a subdirectly irreducible MV-algebra, resp. a subdirectly irreducible state-MV-algebra.

\section{References}

1. S. Pulmannová, E. Vinceková: MV-pair and state operator, Fuzzy Sets and Sysrtems,260 (2015), 62-76.

2. D. Buhagiar, E. Chetcuti, A. Dvurečenskij: Loomis-Sikorski theorem and Stone duality for effect algebras with internal state, Fuzzy Sets and Systems 172 (2011), 71-86.

3. C.C. Chang: Algebraic analysis of many-valued logics, Trans. amer. Math. Soc. 88 (1958), 467-490. 
4. F. Chovanec, F. Kôpka: Boolean D-posets, Tatra Mountains Math. Publ. 10 (1997), 1-15.

5. R.L. Cignoli, I.M. D’Ottaviano, D. Mundici: Algebraic Foundations of ManyValued Reasoning. Springer, 2000 edition, 1999.

6. A. Di Nola, A. Dvurečenskij: State-morphism MV-algebras, J. Pure Appl. Logic 161 (2009), 161-173.

7. A. Di Nola, M. Holčapek, G. Jenča: The category of $M V$-pairs, Logic Journal of the IGLP 17 (2009), 395-412.

8. T. Flaminio, F. Montagna: $M V$-algebras with internal states and probabilistic fuzzy logics, International Journal of Approximate Reasoning 50 (2009), 138-152.

9. D.J. Foulis, M.K. Bennett: Effect algebras and unsharp quantum logics, Found. Phys. 24 (1994), 1325-1346.

10. G. Jenča: A representation theorem for MV-algebras, Soft Computing 11 (2007), 557-564.

11. G. Jenča: Boolean algebras R-generated by $M V$-effect algebras, Fuzzy sets and systems 145 (2004), 279-285.

12. D. Mundici: Averaging the truth-value in Eukasziewicz logic, Studia Logica $\mathbf{5 5}$ (1995), 113-127.

13. T. Vetterlein: Boolean algebras with an automorphism group: a framework for Eukasziewicz logic, J. Multi-Valued log. Soft Comput. 14 (2008), 51-67. 